OPEN ACCESS

Edited by:

Boris Zhivotovsky,

Karolinska Institute (KI), Sweden

Reviewed by:

Daniel Christian Hoessli,

University of Karachi, Pakistan

*Correspondence:

Rong Jiang

Jiangrong1989@sina.com

Juncheng Zhang

zhang.juncheng@sanmedbio.com

tThese authors have contributed equally to this work

Specialty section:

This article was submitted to Molecular and Cellular Oncology, a section of the journa Frontiers in Oncology

Received: 22 August 2019 Accepted: 06 September 2019 Published: 25 September 2019

Citation:

Ma C, Huang C, Tang D, Ye X, Li Z Liu R, Mu N, Li J, Jiang R and Zhang J (2019) Corrigendum: Afatinib for

Advanced Non-small Cell Lung

Cancer in a Case With an Uncommon Epidermal Growth Factor Receptor

Mutation (G719A) Identified in the Cerebrospinal Fluid.

Front. Oncol. 9:938.

doi: 10.3389/fonc.2019.00938

\section{Corrigendum: Afatinib for Advanced Non-small Cell Lung Cancer in a Case With an Uncommon Epidermal Growth Factor Receptor Mutation (G719A) Identified in the Cerebrospinal Fluid}

\author{
Chunhua Ma ${ }^{1 \dagger}$, Chuoji Huang ${ }^{2,3+}$, Dongjiang Tang ${ }^{2,3}$, Xin Ye ${ }^{2,3}$, Zhi Li ${ }^{4}$, Renzhong Liu ${ }^{4}$, \\ Ning $\mathrm{Mu}^{1}$, Jing $\mathrm{Li}^{1}$, Rong Jiang ${ }^{1 * t}$ and Juncheng Zhang $^{2,3 *}$ \\ ${ }^{1}$ Department of Intervention, Tianjin Huanhu Hospital, Tianjin Key Laboratory of Cerebral Vascular and Neurodegenerative \\ Disease, Tianjin, China, ${ }^{2}$ Zhuhai SanMed Biotech Ltd., Zhuhai, China, ${ }^{3}$ Joint Research Center of Liquid Biopsy in \\ Guangdong, Hong Kong and Macao, Zhuhai, China, ${ }^{4}$ Zhuhai Livzon Gene Diagnostics Ltd., Zhuhai, China
}

Keywords: cerebrospinal fluid, G719A mutation, leptomeningeal metastases, NSCLC, afatinib

\section{A Corrigendum on}

Afatinib for Advanced Non-small Cell Lung Cancer in a Case With an Uncommon Epidermal Growth Factor Receptor Mutation (G719A) Identified in the Cerebrospinal Fluid by Ma, C., Huang, C., Tang, D., Ye, X., Li, Z., Liu, R., et al. (2019). Front. Oncol. 9:628. doi: $10.3389 /$ fonc.2019.00628

In the original article, there was an error. "G719A" was erroneously written as "G791A."

A correction has been made the Case Report section, paragraph four:

"After discharge, the patient continued to take afatinib ( $30 \mathrm{mg}$ po qd). Headache and dizziness improved substantially, suggesting effective treatment. Follow-up on 25th July 2018 (day 98) demonstrated reduced pulmonary lesions (chest CT; Figure 1) and improved supratentorial ventricular dilation. On 27th July 2018 (day 100), lumbar puncture showed a CSF pressure of $15 \mathrm{mmHg}$ and CSF CEA levels of 2,111 ng/mL. Intrathecal chemotherapy was performed using methotrexate $(8 \mathrm{mg})$ and dexamethasone $(1 \mathrm{~mL})$ in saline $(9 \mathrm{~mL})$. CSF and peripheral blood samples were collected for repeat genetic testing; the pressure of the ventriculoperitoneal shunt device was adjusted to 1.0, with afatinib dose increased to $40 \mathrm{mg}$ qd. The main adverse effects during afatinib therapy were face and trunk rashes, requiring no treatment. On 10th August 2018 (day 114), NGS results for the CSF revealed an EGFR G719A mutation with a frequency of 22.2\% (around $60 \%$ less than pre-afatinib therapy) and a very low frequency (0.6\%) of NRAS G12V mutation, which is associated with drug resistance (Table 1). EGFR G719A mutation was undetectable in peripheral blood, although EGFR G873R mutation was identified at low frequency (Table 1). On 20th November 2018 (day 216), peripheral blood CEA level was $93 \mathrm{ng} / \mathrm{mL}$; CSF CEA level was $1,590 \mathrm{ng} / \mathrm{mL}$ (Figure 2), with a CSF pressure of $6 \mathrm{mmHg}$, indicating maintenance of the response to afatinib therapy. NGS results for the CSF revealed an EGFR G719A mutation frequency of $23.1 \%$, comparable to that measured on 10th August 2018, while liquid biopsy of peripheral blood indicated increased frequency of EGFR G873R mutation to 2.2\% (Table 1). The patient reported intermittent headache likely from meningeal metastasis. However, the patient had good clinical condition (KPS score, 70-80 points). At last follow-up (1st December 2018; day 227), the patient 
remained in good condition. Although the patient reported mild rash, diarrhea, and oral mucositis, no serious adverse reactions to afatinib had occurred. Thus, the patient had progression-free survival (PFS) > 7 months."

The authors apologize for this error and state that this does not change the scientific conclusions of the article in any way. The original article has been updated.
Copyright (c) 2019 Ma, Huang, Tang, Ye, Li, Liu, Mu, Li, Jiang and Zhang. This is an open-access article distributed under the terms of the Creative Commons Attribution License (CC BY). The use, distribution or reproduction in other forums is permitted, provided the original author $(s)$ and the copyright owner(s) are credited and that the original publication in this journal is cited, in accordance with accepted academic practice. No use, distribution or reproduction is permitted which does not comply with these terms. 